\title{
Micro-Macro limit of a non-local generalized Aw-Rascle type model
}

\author{
Felisia Angela Chiarello ${ }^{1,3} \quad$ Jan Friedrich $^{2} \quad$ PaOla Goatin $^{1}$ \\ Simone GÖTtLich ${ }^{2}$
}

January 16, 2020

\begin{abstract}
We introduce a Follow-the-Leader approximation of a non-local generalized Aw-Rascle-Zhang (GARZ) model for traffic flow. We prove the convergence to weak solutions of the corresponding macroscopic equations deriving $\mathbf{L}^{\infty}$ and BV estimates. We also provide numerical simulations illustrating the micro-macro convergence and we investigate numerically the non-local to local limit for both the microscopic and macroscopic models.
\end{abstract}

Key words: traffic flow, second-order models, non-local conservation laws, micro-macro limits. AMS classification: $35 \mathrm{~L} 65,65 \mathrm{M} 12,90 \mathrm{~B} 20$

\section{Introduction}

The first macroscopic traffic model was introduced more than sixty years ago by Lighthill, Whitham and Richards (LWR) in [22, 23]. Since then, different ways to describe traffic flow phenomena have been developed, using microscopic, mesoscopic and macroscopic modelling approaches.

In this work, we will consider generalizations of the LWR model. This is a first-order macroscopic model consisting of one scalar hyperbolic equation, that expresses the conservation of mass. One well-known extension is the Aw-Rascle-Zhang (ARZ) model [2, 26], that is a macroscopic second-order model, consisting of two hyperbolic equations expressing the conservation of mass and momentum. In contrast to first order traffic flow models, second-order models consider different velocity curves for different drivers. The ARZ second-order model has been improved in recent years by the generalized Aw-Rascle-Zhang (GARZ) model [13]. The GARZ model is characterized by a general relation between the traffic density and the empty road velocity. In particular, unlike the classical ARZ model, one can define this relation in such a way that all drivers have a unique maximum density at which they stop.

Other extensions of the LWR model involve non-local speed dependencies. In recent years, nonlocal conservation laws have become of interest in modelling traffic flow $[3,5,6,14]$. These models take into account the look-ahead distance of drivers, such that vehicles adapt their speed with respect to the downstream traffic. In literature, two different modelling approaches are proposed: either drivers react to the mean downstream traffic density $[3,6]$, or to the mean downstream velocity $[5,14]$. The well-posedness of these models is proved in $[6,14]$.

Besides, the corresponding non-local microscopic models are investigated in [7, 16, 24]. In particular, [16] provides a rigorous micro-macro convergence proof, while [7, 24] analyze the convergence of travelling waves and stationary wave profiles to the macroscopic models.

Microscopic traffic flow models are based on modelling each individual vehicle trajectory via a system of Ordinary Differential Equations (ODEs). First order microscopic models are considered

\footnotetext{
${ }^{1}$ Inria Sophia Antipolis - Méditerranée, Université Côte d'Azur, Inria, CNRS, LJAD, 2004 route des Lucioles BP 93, 06902 Sophia Antipolis Cedex, France. E-mail: paola.goatin@inria.fr

${ }^{2}$ University of Mannheim, Germany, E-mail: $\{j$ an.friedrich, goettlich\}@uni-mannheim.de

${ }^{3}$ Current affiliation: Politecnico di Torino, Department of Mathematical Sciences "G. L. Lagrange", Corso Duca degli Abruzzi 24, 10129 Torino, Italy. E-mail: felisia.chiarello@polito.it
} 
in $[9,15]$ and a second order microscopic model is introduced in [1] with a formal proof of its limit. The rigorous proofs of convergence of the microscopic models to their macroscopic equivalent have been obtained more recently, see $[18,12]$ for the LWR model and [11] for the ARZ model.

In this paper, we introduce a microscopic second order model with non-local interaction, we derive the corresponding non-local macroscopic equations, resulting in a non-local GARZ model, and we prove rigorously the micro-macro convergence. The paper is organized as follows. In Section 2, we describe a non-local microscopic second order model and we provide some relevant estimates and properties. After that, we derive the corresponding macroscopic model in Section 3. Then, in Section 4, we prove the convergence of the microscopic model to the macroscopic one. Finally, we provide numerical tests illustrating the micro-macro convergence and analyzing the limit models (micro and macro) as the look-ahead distance tends to zero.

\section{Non-local GARZ follow-the-leader model}

Non-local microscopic traffic flow models were introduced in $[16,24]$. In these non-local FtL (Followthe-Leader) models the speed of the $i$-th car depends on a weighted mean downstream velocity or downstream density, where the average is computed over an interval of length $\eta$ in front of the $i$-th car. Here, we focus on the model with a weighted downstream velocity [24], which reads

$$
\left\{\begin{array}{l}
\dot{x}_{i}(t)=\sum_{j=0}^{N_{\eta}-1} \gamma_{i, j}(t) v\left(\frac{1}{N\left(x_{i+j+1}(t)-x_{i+j}(t)\right.}\right), \quad i=0, \ldots, N-1 \\
\dot{x}_{N}(t)=v_{\max }
\end{array}\right.
$$

where $x_{i}(t)$ represents the position of the $i$-th car at time $t, N+1$ is the total amount of cars and $1 /(N+1)$ can be interpreted as the length of each car. In addition, $v(\cdot) \in \mathbf{C}^{2}$ is a velocity function satisfying

$$
v(0)=v_{\max }, v\left(\rho_{\max }\right)=0 \text { and } v^{\prime}(\rho)<0 \text { for } \rho \in\left[0, \rho_{\max }\right] .
$$

Formally the microscopic model (2.1) corresponds to the following macroscopic equation:

$$
\partial_{t} \rho+\partial_{x}\left(\rho \int_{x}^{x+\eta} K_{\eta}(y-x) v(\rho(t, y)) d y\right)=0,
$$

for $\eta>0$ and the following assumptions on the kernel function $K_{\eta}$ :

$$
\left.K_{\eta}^{\prime}(x) \leq 0 \text { and continuous on }\right] 0, \eta\left[, \quad \int_{0}^{\eta} K_{\eta}(x) d x=1, \quad K_{\eta}(x)=0 \forall x \notin[0, \eta] .\right.
$$

The relation between the microscopic weights $\gamma_{i, j}$ and the kernel function $K_{\eta}$ is expressed by the following equation:

$$
\gamma_{i, j}(t):= \begin{cases}\int_{x_{i+j}(t)}^{x_{i+j}(t)} K_{\eta}\left(y-x_{i}(t)\right) d y, & \text { if } i+j \leq N-1, \\ \int_{\min \{(t)+\eta}^{x_{i}\left(x_{N}(t), x_{i}(t)+\eta\right\}} K_{\eta}\left(y-x_{i}(t)\right) d y, & \text { if } i+j=N, \\ 0, & \text { if } i+j>N,\end{cases}
$$

with the abuse of notation that $\int_{a}^{a} K_{\eta}(y) d y=0$. In addition $N_{\eta}$ in (2.1) is chosen in such a way that $x_{i+N_{\eta}}-x_{i} \geq \eta$, e.g. $N_{\eta}$ can be the smallest integer such that $N_{\eta} \geq(N+1) \eta$. Note that the weights can be zero for $j$ large (even if $i+j \leq N$ ). 
Our aim is to extend the first order microscopic model (2.1) and to derive a second order model. Therefore we introduce the Lagrangian marker $\omega$ for each individual as a new variable into the velocity function. The Lagrangian marker can be interpreted as an empty road velocity, which might differ depending on individual driver types [13]. Here the classical Aw-Rascle-Zhang (ARZ) model is extended by choosing a more general velocity function, obtaining the so called generalized ARZ (GARZ) model.

We consider the following assumptions on the velocity function $V(\rho, \omega)$, as in [13]:

$$
\begin{aligned}
& V(\rho, \omega) \geq 0, \quad V(0, \omega)=\omega, \quad V(\rho, 0)=0, \\
& \text { for } Q(\rho, \omega)=\rho V(\rho, \omega) \text { we have } \frac{\partial^{2} Q}{\partial \rho^{2}}(\rho, \omega)<0 \text { for } \omega>0, \\
& \frac{\partial V}{\partial \omega}(\rho, \omega)>0 .
\end{aligned}
$$

As stated in [13], the first assumption in (2.4a) ensures that vehicles never travel backwards, while the second one shows why $\omega$ can be interpreted as the empty road velocity. (2.4b) implies $\frac{\partial V}{\partial \rho}(\rho, \omega)<0$ for $\omega>0$, if $V$ is a $C^{2}$ function in $\rho$, see also [13, Lemma 1]. The assumption (2.4c) means that a faster empty road velocity results in a faster velocity for all possible densities.

The main reason of introducing this more general velocity functions is to overcome a shortcoming of the ARZ model. In fact, this latter model has a family of velocity curves and there is not a unique maximum density at which the velocities are zero. Since the maximum density is a property of the road, it should not depend on the velocity assumed by drivers. The GARZ model generalizes the relations between density, Lagrangian marker and velocity, in order to ensure a unique maximum density. In this way, we can choose a velocity function, for which there exists a unique maximum density $R$, such that all cars stop at this density independently of their empty road velocity, i.e.

$$
\exists R>0: \quad V(R, \omega)=0, \quad \forall \omega>0 .
$$

During our work we will assume (2.4) and instead of (2.5) a weaker assumption, namely

$$
\forall \omega>0 \quad \exists R_{\omega}>0: \quad V\left(R_{\omega}, \omega\right)=0 .
$$

Inspired by the first order microscopic equations in (2.1) and introducing a constant in time Lagrangian marker for each individual driver $\omega_{i}$ (see also [11]), we consider the following microscopic equations for $N+1$ vehicles to recover a second order model:

$$
\begin{cases}\dot{x}_{i}(t)=\sum_{j=0}^{N_{\eta}-1} \gamma_{i, j}(t) V_{i, j}(t), & i=0, \ldots, N, \\ \dot{\omega}_{i}(t)=0, & i=0, \ldots, N,\end{cases}
$$

where we define the velocity $V_{i, j}$ as follows

$$
V_{i, j}(t)= \begin{cases}V\left(\frac{1}{N\left(x_{i+1+j}(t)-x_{i+j}(t)\right)}, \omega_{i+j}(t)\right), & \text { for } i+j \leq N-1 \\ V\left(0, \omega_{N-1}(t)\right)=\omega_{N-1}(t), & \text { for } i+j>N-1,\end{cases}
$$

with the weights defined as in (2.3) and the assumptions (2.4) and (2.6) on $V$. For some strictly positive constants $\omega_{0}, \ldots, \omega_{N}$, we can write the model also as follows

$$
\dot{x}_{i}(t)=\sum_{j=0}^{N_{\eta}-1} \gamma_{i, j}(t) V_{i, j}(t), \quad i=0, \ldots, N
$$


and the velocity $V_{i, j}$ simplifies to

$$
V_{i, j}(t)= \begin{cases}V\left(\frac{1}{N\left(x_{i+1+j}(t)-x_{i+j}(t)\right)}, \omega_{i+j}\right), & \text { for } i+j \leq N-1 \\ \omega_{N-1}, & \text { for } i+j>N-1 .\end{cases}
$$

In the following we will show some properties of the microscopic model (2.8). These properties are necessary to prove the convergence to the macroscopic model derived in Section 3.

\subsection{Construction of initial positions}

In order to solve the microscopic equation (2.7) (and also (2.1)) initial vehicle positions $x_{0}(0)<$ $\cdots<x_{N}(0)$ have to be prescribed. We define $x_{i}^{0}:=x_{i}(0)$.

Since we are interested in the convergence to a macroscopic model we describe how to create the initial positions for a given initial density $\rho_{0}(x)$. We assume that the initial datum satisfies

$$
\rho_{0}(x) \in B V(\mathbb{R}), \rho_{0}(x) \leq R, \operatorname{supp}\left(\rho_{0}\right) \text { compact and connected. }
$$

For simplicity, we normalize the total initial mass so that

$$
\int_{\mathbb{R}} \rho_{0}(x) d x=1
$$

Now we define moving vehicles, which follow our micro dynamics $(2.7)$. Therefore, we set $\left[x_{\min }^{0}, x_{\max }^{0}\right]$ as the closed convex hull of $\operatorname{supp}\left(\rho_{0}\right)$ and for a fixed integer $N$ we split up this interval into $N$ smaller intervals, such that the integral of $\rho_{0}$ over each interval equals $1 / N$, i.e. $x_{0}^{0}=x_{\min }^{0}$ and $x_{N}^{0}=x_{\max }^{0}$ and we define recursively

$$
x_{i}^{0}=\sup \left\{x \in \mathbb{R}: \int_{x_{i-1}^{0}}^{x} \rho_{0}(x) d x<\frac{1}{N}\right\}, \quad i=1, \ldots, N-1 .
$$

The initial Lagrangian marker for each driver is obtained from an initial condition $\omega_{0}(x)$ by

$$
\omega_{i}^{0}=\underset{\left[x_{i}^{0}, x_{i+1}^{0}\right]}{\operatorname{ess} \sup }\left(\omega_{0}\right), \quad i=0, \ldots, N-1 .
$$

\subsection{Weak Maximum Principle for the GARZ non-local micro-model}

Let us denote by $R_{i}$ the solution to $V\left(R_{i}, \omega_{i}\right)=0$ for a given positive constant $\omega_{i}$. Due to assumption (2.6), $R_{i}$ exists and can be interpreted as the individual maximum discrete density at which driver $i$ comes to stop. In addition, we denote by $L_{i}:=\frac{1}{N R_{i}}$ the individual minimum distance each car keeps to the car in front of it. We will now prove that cars cannot overtake each other.

Proposition 1. Consider a sequence $x_{0}^{0}<x_{1}^{0}<\ldots<x_{N}^{0}$ and denote with $x(t)=\left(x_{0}(t), \ldots, x_{N}(t)\right)$ the solution of (2.8). Let us assume (2.2) and (2.4). If $x_{i+1}^{0}-x_{i}^{0} \geq L_{i}$ for all $i=0, \ldots, N-1$, then it holds $x_{i+1}(t)-x_{i}(t) \geq L_{i}$ for all times $t>0$.

Proof. We prove that if for some $t>0$ there holds $x_{i+1}(t)-x_{i}(t)=L_{i}$ and $x_{j+1}(t)-x_{j}(t) \geq L_{j}$ for $j>i$, then $\dot{x}_{i+1}(t)-\dot{x}_{i}(t) \geq 0$. If $i=N-1$, we have

$$
\dot{x}_{N-1}=\gamma_{N-1,0} V\left(\frac{1}{N\left(x_{N}(t)-x_{N-1}(t)\right)}, \omega_{N-1}\right)+\left(1-\gamma_{N-1,0}\right) \omega_{N-1} \leq \omega_{N-1}=\dot{x}_{N},
$$


since $V(\rho, \omega) \leq \omega$. If $i<N-1$, observing that $V_{i+1, j}(t)=V_{i, j+1}(t)$, we have

$$
\begin{aligned}
\dot{x}_{i+1}(t)-\dot{x}_{i}(t) & =\sum_{j=0}^{N_{\eta}-1} \gamma_{i+1, j}(t) V_{i+1, j}(t)-\sum_{j=0}^{N_{\eta}-1} \gamma_{i, j}(t) V_{i, j}(t) \\
& =\sum_{j=1}^{N_{\eta}-1}\left(\gamma_{i+1, j-1}(t)-\gamma_{i, j}(t)\right) V_{i, j}(t)-\gamma_{i, 0}(t) V_{i, 0}(t)+\gamma_{i+1, N_{\eta}-1}(t) V_{i, N_{\eta}}(t) .
\end{aligned}
$$

Under our hypothesis all coefficients of $V_{i, j}(t)$ are positive and also the velocities. In addition, we have

$$
V_{i, 0}(t)=V\left(\frac{1}{N L_{i}}, \omega_{i}\right)=V\left(R_{i}, \omega_{i}\right)=0
$$

so that we can conclude that

$$
\dot{x}_{i+1}(t)-\dot{x}_{i}(t) \geq 0
$$

Remark 1. If we assume in addition to (2.2) and (2.4) also (2.6), the previous proposition implies that the micro model has a unique maximum density $R$.

\subsection{BV estimates for the GARZ non-local micro-model}

Since we consider microscopic interactions between vehicles, it might be reasonable to assume that the initial density is greater than zero in the interval between the position of the first vehicle and the last one. We need this assumption to prove uniform BV bounds.

Assumption 1. We assume there exists $\rho_{\min }>0$ such that $\rho_{0}(x) \geq \rho_{\min }$ for all $x \in \operatorname{supp}\left(\rho_{0}\right)$.

Lemma 1. Under Assumption 1, we obtain

$$
N\left(x_{i+1}(0)-x_{i}(0)\right) \leq \frac{1}{\rho_{\min }} .
$$

Proof. From the definition of the initial conditions, following the computations in [10]

$$
\begin{aligned}
x_{i+1}(0) & =\sup \left\{x \in \mathbb{R}: \int_{x_{i}(0)}^{x} \rho_{0}(x) d x<\frac{1}{N}\right\} \\
& \leq \sup \left\{x \in \mathbb{R}: \int_{x_{i}(0)}^{x} \rho_{\min } d x<\frac{1}{N}\right\} \\
& \leq \sup \left\{x \in \mathbb{R}:\left(x-x_{i}(0)\right) \rho_{\min }=\frac{1}{N}\right\} \\
& =\frac{1}{N \rho_{\min }}+x_{i}(0) .
\end{aligned}
$$

This gives the desired estimate.

We want to obtain a bound on the distance between two consecutive cars by using Gronwall's inequality. For readability, let us define

$$
R=\max _{i=0, \ldots, N-1} R_{i}, \quad v_{\max }=\max _{i=0, \ldots, N-1} \omega_{i} .
$$


Lemma 2. The following estimate holds for every $t>0$ :

$$
x_{i+1}(t)-x_{i}(t) \leq\left(x_{i+1}(0)-x_{i}(0)\right) \exp \left(t v_{\max } K_{\eta}(0)\right), \quad i=0, \ldots, N-1 .
$$

Proof. We have

$$
\begin{aligned}
\dot{x}_{i+1}(t)-\dot{x}_{i}(t) & =\sum_{j=1}^{N_{\eta}-1}(\underbrace{\gamma_{i+1, j-1}(t)-\gamma_{i, j}(t)}_{\geq 0}) V_{i, j}(t)-\gamma_{i, 0}(t) V_{i, 0}(t)+\gamma_{i+1, N_{\eta}-1}(t) V_{i, N_{\eta}}(t) \\
& \leq v_{\max }\left(\sum_{j=1}^{N_{\eta}-1}\left(\gamma_{i+1, j-1}(t)-\gamma_{i, j}(t)\right)+\gamma_{i+1, N_{\eta}-1}(t)\right) \\
& =v_{\max }\left(\sum_{j=0}^{N_{\eta}-1} \gamma_{i+1, j}(t)-\sum_{j=1}^{N_{\eta}-1} \gamma_{i, j}(t)\right) \\
& =v_{\max }\left(1-\left(1-\gamma_{i, 0}\right)\right) \\
& =v_{\max } \gamma_{i, 0} \\
& =v_{\max } \int_{x_{i}(t)}^{x_{i+1}(t)} K_{\eta}\left(y-x_{i}(t)\right) d y \\
& \leq v_{\max } K_{\eta}(0)\left(x_{i+1}(t)-x_{i}(t)\right)
\end{aligned}
$$

where we used the monotonicity of the kernel function. Applying Gronwall's inequality, we get the statement.

An immediate consequence of the two lemmas above are the following bounds:

$$
\begin{aligned}
& N\left(x_{i+1}(t)-x_{i}(t)\right) \leq \frac{\exp \left(t v_{\max } K_{\eta}(0)\right)}{\rho_{\min }} \\
& N\left(\dot{x}_{i+1}(t)-\dot{x}_{i}(t)\right) \leq K_{\eta}(0) v_{\max } \frac{\exp \left(t v_{\max } K_{\eta}(0)\right)}{\rho_{\min }} .
\end{aligned}
$$

Let us now define the microscopic density as

$$
\rho_{i}^{N}(t):=\frac{1}{N\left(x_{i+1}(t)-x_{i}(t)\right)}, \quad i=0, \ldots, N-1,
$$

and the corresponding piece-wise constant function

$$
\rho^{N}(t, x):=\sum_{i=0}^{N-1} \rho_{i}^{N}(t) \chi_{\left[x_{i}(t), x_{i+1}(t)[\right.}(x) .
$$

Proposition 2. Let $\rho_{0}$ satisfy (2.9) and Assumption 1. Then, for every $N \in \mathbb{N}$ one has

$$
T V\left(\rho^{N}(t, \cdot)\right) \leq T V\left(\rho_{0}\right) \exp \left(\int_{0}^{t} C(\tau) d \tau\right),
$$

where $C(t)=v_{\max } K_{\eta}(0) R \frac{\exp \left(t v_{\max } K_{\eta}(0)\right)}{\rho_{\min }}$, for $T>0$. 
Proof. To simplify the notation, we will drop the index $N$ in $\rho_{i}^{N}(t)$. Following the computations in [10], the total variation is given by

$$
\begin{aligned}
T V\left(\rho^{N}(t, \cdot)\right)= & \rho_{0}(t)+\rho_{N-1}(t)+\sum_{i=0}^{N-2}\left|\rho_{i+1}(t)-\rho_{i}(t)\right| \\
= & \sum_{i=1}^{N-2} \rho_{i}(t) \underbrace{\left(\operatorname{sgn}\left(\rho_{i}(t)-\rho_{i-1}(t)\right)-\operatorname{sgn}\left(\rho_{i+1}(t)-\rho_{i}(t)\right)\right.}_{=: \mu_{i}(t)}+ \\
& \rho_{0}(t) \underbrace{\left(1-\operatorname{sgn}\left(\rho_{1}(t)-\rho_{0}(t)\right)\right)}_{=: \mu_{0}(t)}+\rho_{N-1}(t) \underbrace{\left(1+\operatorname{sgn}\left(\rho_{N-1}(t)-\rho_{N-2}(t)\right)\right.}_{=: \mu_{N-1}(t)} .
\end{aligned}
$$

Therefore, we obtain

$$
\frac{d}{d t} T V\left(\rho^{N}(t, \cdot)\right)=\sum_{i=1}^{N-2} \mu_{i}(t) \dot{\rho}_{i}(t)+\mu_{0}(t) \dot{\rho}_{0}(t)+\mu_{N-1}(t) \dot{\rho}_{N-1}(t) .
$$

By $(2.13)$, we get $\dot{\rho}_{i}(t)=-N \rho_{i}(t)^{2}\left(\dot{x}_{i+1}(t)-\dot{x}_{i}(t)\right)$. Obviously, we have

$$
\mu_{0}(t)= \begin{cases}0, & \text { if } \rho_{1}(t)>\rho_{0}(t) \\ 2, & \text { if } \rho_{1}(t)<\rho_{0}(t) .\end{cases}
$$

In addition, recalling the definition of $\gamma_{0,0}$ given by (2.3) and the monotonicity of the kernel function

$$
\begin{aligned}
\dot{\rho}_{0}(t) & =-N \rho_{0}(t) \rho_{0}(t)\left(\dot{x}_{1}(t)-\dot{x}_{0}(t)\right) \\
& =\rho_{0}(t) \frac{1}{x_{1}(t)-x_{0}(t)}(\sum_{j=1}^{N_{\eta}-1} \underbrace{\left(\gamma_{0, j}(t)-\gamma_{1, j-1}(t)\right)}_{\leq 0} V_{0, j}(t)+\gamma_{0,0}(t) V_{0,0}(t)-\gamma_{1, N_{\eta}-1}(t) V_{0, N_{\eta}}(t)) \\
& \leq \rho_{0}(t) \frac{1}{x_{1}(t)-x_{0}(t)} \gamma_{0,0}(t) V_{0,0}(t) \\
& \leq \rho_{0}(t) K_{\eta}(0) v_{\max } .
\end{aligned}
$$

Similarly, we have

$$
\mu_{N-1}(t)= \begin{cases}0, & \text { if } \rho_{N-1}(t)<\rho_{N-2}(t) \\ 2, & \text { if } \rho_{N-1}(t)>\rho_{N-2}(t)\end{cases}
$$

and

$$
\begin{aligned}
\dot{\rho}_{N-1}(t) & =-N \rho_{N-1}(t) \rho_{N-1}(t)\left(\dot{x}_{N}(t)-\dot{x}_{N-1}(t)\right) \\
& =\frac{\rho_{N-1}(t)}{x_{N}(t)-x_{N-1}(t)}\left(\gamma_{N-1,0}(t) V_{N-1,0}(t)+\left(1-\gamma_{N-1,0}(t)\right) \omega_{N-1}-\omega_{N-1}\right) \\
& =\frac{\rho_{N-1}(t)}{x_{N}(t)-x_{N-1}(t)} \gamma_{N-1,0}(t)\left(V_{N-1,0}(t)-\omega_{N-1}\right) \\
& \leq \rho_{N-1}(t) K_{\eta}(0) v_{\max } .
\end{aligned}
$$

Finally,

$$
\sum_{i=1}^{N-1} \mu_{i}(t) \dot{\rho}_{i}(t)=\sum_{i=1}^{N-2} \mu_{i}(t) \rho_{i}(t) \underbrace{\left(-\rho_{i}(t) N\left(\dot{x}_{i+1}(t)-\dot{x}_{i}(t)\right)\right)}_{=: I_{i}} .
$$


Following the computations above and applying (2.11) and (2.12), we obtain

$$
\begin{aligned}
\left|I_{i}\right| & \leq R_{i} N\left|\dot{x}_{i+1}(t)-\dot{x}_{i}(t)\right| \\
& \leq R_{i} K_{\eta}(0) v_{\max } N\left(x_{i+1}(t)-x_{i}(t)\right) \\
& \leq R_{i} K_{\eta}(0) v_{\max } \frac{\exp \left(t v_{\max } K_{\eta}(0)\right)}{\rho_{\min }}
\end{aligned}
$$

which gives

$$
\frac{d}{d t} T V\left(\rho^{N}(t, \cdot)\right) \leq C(t) T V\left(\rho^{N}(t, \cdot)\right)
$$

with

$$
C(t)=v_{\max } K_{\eta}(0) R \frac{\exp \left(t v_{\max } K_{\eta}(0)\right)}{\rho_{\min }} .
$$

Applying Gronwall's inequality, we get the desired BV estimate.

Remark 2. The proof of the maximum principle and the $B V$ estimates are also valid in the case $\omega_{i}=\omega \forall i=0, \ldots, N-1$. This case corresponds to the first order setting of [24]. Therefore, following the proof in section 4, an analogous convergence result can be obtained for the first order non-local microscopic model (2.1).

Before giving the rigorous convergence proof in section 4, we derive the corresponding macroscopic model.

\section{Formal derivation from microscopic equations}

In the following, we formally derive the macroscopic system corresponding to the microscopic dynamics (2.7). We will refer to the resulting macro model as "non-local GARZ model". This is given by

$$
\left\{\begin{array}{l}
\rho_{t}+\left(\rho\left(K_{\eta} * V(\rho, \omega)\right)\right)_{x}=0 \\
\omega_{t}+\left(K_{\eta} * V(\rho, \omega)\right) \omega_{x}=0,
\end{array}\right.
$$

where $K_{\eta} * V(\rho, \omega)(t, x):=\int_{x}^{x+\eta} K_{\eta}(y-x) V(\rho(t, y), \omega(t, y)) d y$.

Setting $q=\rho \omega$, the model can be written in conservative form for $\rho \neq 0$ as

$$
\left\{\begin{array}{l}
\rho_{t}+\left(\rho\left(K_{\eta} * V\left(\rho, \frac{q}{\rho}\right)\right)\right)_{x}=0 \\
q_{t}+\left(q\left(K_{\eta} * V\left(\rho, \frac{q}{\rho}\right)\right)\right)_{x}=0,
\end{array}\right.
$$

where the convolution product is defined as above.

Since the derivation of such macroscopic equations is not unique and straightforward, we present our approach below, following mainly the ideas presented in $[4,19]$. 


\subsection{From microscopic model to macroscopic model in Lagrangian coordinates}

In addition to the microscopic density (2.13), we define

$$
y_{i}(t)=\frac{1}{\rho_{i}(t)}=N\left(x_{i+1}(t)-x_{i}(t)\right) .
$$

Now let us consider the weights defined in (2.3) for the case $i+j \leq N-1$, where we have

$$
\gamma_{i, j}(t)=\int_{x_{i+j}(t)-x_{i}(t)}^{x_{i+j+1}(t)-x_{i}(t)} K_{\eta}(y) d y
$$

By having a closer look at the difference of $x_{i+j}$ and $x_{i}$ we obtain

$$
x_{i+j}(t)-x_{i}(t)=\sum_{k=0}^{j-1} x_{i+j-k}(t)-x_{i+j-k-1}(t)=\sum_{k=0}^{j-1} \frac{1}{N \rho_{i+j-k-1}(t)} .
$$

Now we define the discretization for $z$, the Lagrangian coordinate, as $z_{i}=\frac{i}{N} \in[0,1], i=0, \ldots, N$, and the mapping

$$
\rho(t, z)=\sum_{i=0}^{N-1} \rho_{i}(t) \chi_{\left[z_{i}, z_{i+1}[\right.}(z),
$$

in order to derive the limit of this piecewise constant function as in [19]. In this setting, $z$ represents the continuous number of a car and so $\rho(t, z)$ the density at the car $z$. In addition $\rho(t, z)=0$ for $z \geq 1$. Therefore, we have

$$
\sum_{k=0}^{j-1} \frac{1}{N \rho_{i+j-k-1}(t)}=\sum_{k=0}^{j-1} \int_{z_{i}+(j-k-1) \frac{1}{N}}^{z_{i}+(j-k) \frac{1}{N}} \frac{1}{\rho(t, z)} d z=\int_{z_{i}}^{z_{i}+j \frac{1}{N}} \frac{1}{\rho(t, z)} d z .
$$

We define now the Lagrangian marker and the velocity with respect to the Lagrangian coordinate $z$, i.e.

$$
\tilde{\omega}(t, z)= \begin{cases}\omega(t, x(t, z)) & \text { if } z \in\left[0,1\left[, \quad \tilde{V}(t, z)=\left\{\begin{array}{ll}
V(\rho(t, z), \omega(t, z)) & \text { if } z \in[0,1[, \\
\omega(t, x(t, 1)), & \text { if } z \geq 1,
\end{array} \text { if } z \geq 1 .\right.\right.\right.\end{cases}
$$

Using this we obtain

$$
\sum_{j=0}^{N_{\eta}-1} \gamma_{i, j}(t) V_{i, j}(t)=\sum_{j=0}^{\min \left\{N-1-i, N_{\eta}-1\right\}} \gamma_{i, j}(t) V_{i, j}(t)+\gamma_{i, N-i}(t) V_{i, N-i}(t) .
$$

We note that if $N-i \geq N_{\eta}$, then $N-i \geq \eta N$ and therefore $x_{N}(t)-x_{i}(t) \geq \eta$ and $\gamma_{i, N-i}(t)=0$. Now we will deal with the first term:

$$
\begin{aligned}
& \min \left\{N-1-i, N_{\eta}-1\right\} \\
& \sum_{j=0} \gamma_{i, j}(t) V_{i, j}(t) \\
& =\sum_{j=0}^{\min \left\{N-1-i, N_{\eta}-1\right\}} \tilde{V}\left(t, z_{i}+j \frac{1}{N}\right) \int_{\int_{z_{i}}^{z_{i}+j \frac{1}{N}} \frac{1}{\rho(t, z)}}^{\int_{z_{i}+(j+1) \frac{1}{N}}^{z^{2}} \frac{1}{\rho(t, z)} d z} K_{\eta}(y) d y
\end{aligned}
$$




$$
\begin{aligned}
& =\sum_{j=0}^{\min \left\{N-1-i, N_{\eta}-1\right\}} \tilde{V}\left(t, z_{i}+j \frac{1}{N}\right) \int_{z_{i}+j \frac{1}{N}}^{z_{i}+(j+1) \frac{1}{N}} \frac{1}{\rho(t, y)} K_{\eta}\left(\int_{z_{i}}^{y} \frac{1}{\rho(t, s)} d s\right) d y \\
& =\int_{z_{i}}^{\min \left\{1, z_{i+N_{\eta}}\right\}} \frac{1}{\rho(t, y)} K_{\eta}\left(\int_{z_{i}}^{y} \frac{1}{\rho(t, s)} d s\right) \tilde{V}(t, y) d y,
\end{aligned}
$$

where $z_{i+N_{\eta}}$ is defined as above as $z_{i+N_{\eta}}=\frac{i+N_{\eta}}{N}$, so it can be greater 1 and represents the number of the car, which is at least $\eta$ away, if such a car exists. The second term can be written as

$$
\begin{aligned}
\gamma_{i, N-i}(t) V_{i, N-i}(t) & =\int_{\min \left\{x(t, 1)-x\left(t, z_{i}\right), \eta\right\}}^{\eta} K_{\eta}(y) d y \tilde{V}\left(t, z_{i}+\frac{N-i}{N}\right) \\
& =\int_{\min \left\{x(t, 1)-x\left(t, z_{i}\right), \eta\right\}}^{\eta} K_{\eta}(y) \tilde{V}(t, 1) d y \\
& =\int_{\min \left\{x(t, 1)-x\left(t, z_{i}\right), \eta\right\}}^{\eta} K_{\eta}(y) V(0, \omega(t, y(t, 1))) d y
\end{aligned}
$$

where $x$ is the corresponding Eulerian coordinate of car $z$.

From the microscopic system (2.7), it follows that

$$
\left\{\begin{array}{l}
\dot{y}_{i}(t)=N \sum_{j=0}^{N_{\eta}-1}\left(\gamma_{i+1, j}(t) V_{i+1, j}(t)-\gamma_{i, j}(t) V_{i, j}(t)\right) \\
\dot{w}_{i}(t)=0 .
\end{array}\right.
$$

Passing to the limit $N \rightarrow+\infty$, we obtain that this is only a rough semi-discretization of the following

$$
\left\{\begin{aligned}
\partial_{t} y(t, z)= & \partial_{z}\left(\int_{z}^{\min \left\{1, z_{\eta}\right\}} \frac{1}{\rho(t, y)} K_{\eta}\left(\int_{z}^{y} \frac{1}{\rho(t, s)} d s\right) V(\rho(t, y), \omega(t, y)) d y\right. \\
& \left.+\int_{\min \left\{x(t, 1)-x\left(t, z_{i}\right), \eta\right\}}^{\eta} K_{\eta}(y) V(0, \omega(t, y(t, 1))) d y\right) \\
\partial_{t} w(t, z)= & 0 .
\end{aligned}\right.
$$

Using again the substitution rule and recalling that for an initial condition of compact support the density is zero outside and the Lagrangian marker should be constant to its last value, when the compact support of $\rho$ ends, we get

$$
\left\{\begin{array}{l}
\partial_{t} y(t, z)=\partial_{z} \int_{x(t, z)}^{x(t, z)+\eta} K_{\eta}(y-x(t, z)) V(\rho(t, z(t, y)), \omega(t, z(t, y))) d y \\
\partial_{t} w(t, z)=0
\end{array}\right.
$$

where we used the following relation for the substitution

$$
\partial_{z} x=\frac{1}{\rho(t, z)}
$$

\subsection{From Lagrangian to Eulerian coordinates}

For brevity, let us define $\nu:=K_{\eta} * u$. We can transform the macroscopic system (3.3) that we obtained in Lagrangian coordinates $(t, z)$ to Eulerian coordinates $\left(t^{\prime}, x\right)$ with

$$
\partial_{z} x=y, \quad \partial_{z} t^{\prime}=0, \quad \partial_{t} x=\nu, \quad \partial_{t} t^{\prime}=1 .
$$

Then, we obtain

$$
\partial_{t} y\left(t^{\prime}, x\right)=\partial_{t^{\prime}} y \partial_{t} t^{\prime}+\partial_{x} y \partial_{t} x=\partial_{t^{\prime}} y+\partial_{x} y \nu
$$




$$
\begin{aligned}
& \partial_{z} v\left(t^{\prime}, x\right)=\partial_{t^{\prime}} \nu \partial_{z} t^{\prime}+\partial_{x} \nu \partial_{z} x=\partial_{x} \nu y, \\
& \partial_{t} \omega\left(t^{\prime}, x\right)=\partial_{t^{\prime}} \omega \partial_{t} t^{\prime}+\partial_{x} \omega \partial_{t} x=\partial_{t^{\prime}} \omega+\partial_{x} \omega \nu
\end{aligned}
$$

Observing that

$$
\rho\left(t^{\prime}, x\right)\left(\partial_{t^{\prime}} \omega+\partial_{x} \omega \nu\right)+\omega\left(t^{\prime}, x\right)\left(\partial_{t^{\prime}} \rho+\partial_{x}(\rho \nu)\right)=\partial_{t^{\prime}}\left(\rho\left(t^{\prime}, x\right) \omega\left(t^{\prime}, x\right)\right)+\partial_{x}\left(\rho\left(t^{\prime}, x\right) \nu\left(t^{\prime}, x\right) \omega\left(t^{\prime}, x\right)\right)
$$

and $t^{\prime}=t$, we get the following Eulerian system

$$
\left\{\begin{array}{l}
\partial_{t} \rho+\partial_{x}(\rho \nu)=0 \\
\partial_{t}(\rho \omega)+\partial_{x}(\rho \omega \nu)=0
\end{array}\right.
$$

which is equivalent to (3.2).

\section{Convergence}

In the following we show that the micro dynamics (2.7) converges to a weak solution of the macroscopic equation (3.2). Weak solutions are intended in the following sense.

Definition 1 (Weak solutions). Let $\left(\rho^{0}, q^{0}\right) \in B V(\mathbb{R})$. We say that a couple $(\rho, q)$ of functions $\rho, q \in \mathbf{L}^{\infty}\left(\left[0,+\infty[\times \mathbb{R}) \cap \mathbf{C}\left(\left[0, \infty\left[; \mathbf{L}^{\mathbf{1}}(\mathbb{R})\right)\right.\right.\right.\right.$ is a weak solution of $(3.2)$ if, for any test function $\varphi \in \mathbf{C}_{\mathbf{c}}^{\infty}([0,+\infty[\times \mathbb{R} ; \mathbb{R})$ and $\eta>0$, it satisfies

$$
\int_{\mathbb{R}^{+}} \int_{\mathbb{R}}\left(\varphi_{t}+\left(K_{\eta} * V\left(\rho, \frac{q}{\rho}\right)\right) \varphi_{x}\right) \cdot\left(\begin{array}{l}
\rho \\
q
\end{array}\right) d x d t+\int_{\mathbb{R}} \varphi(0, x) \cdot\left(\begin{array}{l}
\rho^{0} \\
q^{0}
\end{array}\right) d x=\left(\begin{array}{l}
0 \\
0
\end{array}\right) .
$$

In addition to the microscopic density (2.14) let us define analogous functions for the Lagrangian marker and the conserved variable $q$ :

$$
\omega^{N}(t, x)=\sum_{i=0}^{N-1} \omega_{i} \chi_{\left[x_{i}(t), x_{i+1}(t)[\right.}(x) \quad \text { and } \quad q^{N}(t, x)=\sum_{i=0}^{N-1} \omega_{i} \rho_{i}(t) \chi_{\left[x_{i}(t), x_{i+1}(t)[\right.}(x) .
$$

Theorem 1. For any $N \in \mathbb{N}$, let $\left(\rho^{N}, q^{N}\right)$ be defined as in (2.14) and (4.2) and let $\eta>0$ be a fixed value independent of $N$. Then $\left(\rho^{N}, q^{N}\right)$ converges up to a subsequence in $\mathbf{C}\left(\left[0,+\infty\left[; \mathbf{L}^{\mathbf{1}}(\mathbb{R})\right)\right.\right.$ to a weak solution $(\rho, q)$ of $(3.2)$ coupled with an initial datum $\left(\rho^{0}, q^{0}\right) \in B V(\mathbb{R})$.

Proof. For readability, we introduce the notation $D^{+} h_{i}=h_{i+1}-h_{i}$. Observe that $\rho^{N}$ is in $\mathbf{L}^{\mathbf{1}}(\mathbb{R})$, since it is positive and

$$
\frac{d}{d t} \int_{\mathbb{R}} \rho^{N}(t, x) d x=\sum_{i=0}^{N-1} \frac{d}{d t} \int_{x_{i}(t)}^{x_{i+1}(t)} \rho_{i}(t) d x=\sum_{i=0}^{N-1} \frac{d}{d t}\left(\left(x_{i+1}(t)-x_{i}(t)\right) \rho_{i}(t)\right)=0,
$$

due to the definition of $\rho_{i}$ in (2.13).

Let $\varphi=\varphi(t, x)$ be a smooth test function with compact support in $\left[0,+\infty\left[\times \mathbb{R}\right.\right.$. Defining $\varphi\left(t, x_{i}\right)=$ $\varphi_{i}(t)$, we can compute

$$
\int_{\mathbb{R}^{+}} \int_{\mathbb{R}} \rho^{N}(t, x) \varphi_{t}(t, x) d x d t+\int_{\mathbb{R}} \rho^{N}(0, x) \varphi(0, x) d x
$$




$$
\begin{aligned}
& =\int_{\mathbb{R}^{+}} \sum_{i=0}^{N-1} \rho_{i}(t) \int_{x_{i}(t)}^{x_{i+1}(t)} \varphi_{t}(t, x) d x d t+\int_{\mathbb{R}^{\prime}} \rho^{N}(0, x) \varphi(0, x) d x \\
& =\int_{\mathbb{R}^{+}} \sum_{i=0}^{N-1}\left[\rho_{i}(t) \frac{d}{d t}\left(\int_{x_{i}(t)}^{x_{i+1}(t)} \varphi(t, x) d x\right)-\rho_{i}(t) D^{+}\left(\dot{x}_{i}(t) \varphi_{i}(t)\right)\right] d t+\int_{\mathbb{R}^{\prime}} \rho^{N}(0, x) \varphi(0, x) d x \\
& =-\int_{\mathbb{R}^{+}}\left[\sum_{i=0}^{N-1} \int_{x_{i}(t)}^{x_{i+1}(t)} \dot{\rho}_{i}(t) \varphi(t, x) d x-\sum_{i=0}^{N-2} D^{+}\left(\rho_{i}(t)\right) \dot{x}_{i+1}(t) \varphi_{i+1}(t)\right. \\
& \left.\quad+\rho_{N-1}(t) \dot{x}_{N}(t) \varphi_{N}(t)-\rho_{0}(t) \dot{x}_{0}(t) \varphi_{0}(t)\right] d t .
\end{aligned}
$$

Observing that $\dot{\rho}_{i}(t)=-\rho_{i}^{2}(t) N\left(\dot{x}_{i+1}(t)-\dot{x}_{i}(t)\right)$, we can write

$$
\begin{aligned}
(4.3)=\int_{\mathbb{R}^{+}} & \sum_{i=0}^{N-1}\left[\int_{x_{i}(t)}^{x_{i+1}(t)} \rho_{i}^{2}(t) N\left(\dot{x}_{i+1}(t)-\dot{x}_{i}(t)\right) \varphi(t, x) d x\right. \\
& \left.+\sum_{i=0}^{N-2} D^{+}\left(\rho_{i}(t)\right) \dot{x}_{i+1}(t) \varphi_{i+1}(t)-\rho_{N-1}(t) \dot{x}_{N}(t) \varphi_{N}(t)+\rho_{0}(t) \dot{x}_{0}(t) \varphi_{0}(t)\right] d t .
\end{aligned}
$$

We note that for $\varphi(t, x)$ with compact support in $] 0,+\infty[\times \mathbb{R}$ the above equation simplifies to

$$
\int_{\mathbb{R}^{+}} \int_{\mathbb{R}} \rho^{N}(t, x) \varphi_{t}(t, x) d x d t=(4.4) .
$$

Choosing $\varphi(t, x)=\chi_{\left[t_{1}, t_{2}\right]}(t) \psi(x)$ for $0<t_{1}<t_{2}<+\infty$ and a smooth test function $\psi$ with $|\psi| \leq 1$, let $\psi_{i+1}=\psi\left(x_{i+1}\right)$, using (4.3)-(4.4), we can write

$$
\begin{aligned}
& \left|\int_{\mathbb{R}}\left(\rho^{N}\left(t_{2}, x\right)-\rho^{N}\left(t_{1}, x\right)\right) \psi(x) d x\right| \\
= & \mid \int_{t_{1}}^{t_{2}}\left[\sum_{i=0}^{N-1} \int_{x_{i}(t)}^{x_{i+1}(t)} \rho_{i}^{2}(t) N\left(\dot{x}_{i+1}(t)-\dot{x}_{i}(t)\right) \psi(x) d x+\sum_{i=0}^{N-2} D^{+}\left(\rho_{i}(t)\right) \dot{x}_{i+1}(t) \psi_{i+1}\right. \\
- & \left.\rho_{N-1}(t) \dot{x}_{N}(t) \psi_{N}+\rho_{0}(t) \dot{x}_{0}(t) \psi_{0}\right] d t \mid \\
\leq & \int_{t_{1}}^{t_{2}}\left[\sum_{i=0}^{N-1} \int_{x_{i}(t)}^{x_{i+1}(t)} \rho_{i}^{2}(t) N\left|\dot{x}_{i+1}(t)-\dot{x}_{i}(t)\right||\psi(x)| d x+\sum_{i=0}^{N-2}\left|D^{+}\left(\rho_{i}(t)\right)\right|\left|\dot{x}_{i+1}(t)\right|\left|\psi_{i+1}\right|\right. \\
& \left.+\rho_{N-1}(t)\left|\dot{x}_{N}(t)\right|\left|\psi_{N}\right|+\rho_{0}(t)\left|\dot{x}_{0}(t)\right|\left|\psi_{0}\right|\right] d t \\
\leq & \int_{t_{1}}^{t_{2}}\left[\sum_{i=0}^{N-1} \int_{x_{i}(t)}^{x_{i+1}(t)} \rho_{i}^{2}(t) N\left|\dot{x}_{i+1}(t)-\dot{x}_{i}(t)\right| d x+\sum_{i=0}^{N-2}\left|D^{+}\left(\rho_{i}(t)\right)\right|\left|\dot{x}_{i+1}(t)\right|\right. \\
+ & \rho_{N-1}(t)\left|\dot{x}_{N}(t)+\rho_{0}(t)\right| \dot{x}_{0}(t) \mid d t
\end{aligned}
$$




$$
\begin{aligned}
& \leq \int_{t_{1}}^{t^{2}}\left[\sum_{i=0}^{N-1} \int_{x_{i}(t)}^{x_{i+1}(t)} \rho_{i}(t) C(t) d x+v_{\max }\left(\sum_{i=0}^{N-2}\left|D^{+}\left(\rho_{i}(t)\right)\right|+\rho_{N-1}(t)+\rho_{0}(t)\right)\right] d t \\
& \leq \int_{t_{1}}^{t_{2}} C(t)+v_{\max } T V\left(\rho^{N}(t, \dot{)}) d t\right. \\
& \leq\left(C\left(t_{2}\right)+v_{\max } T V\left(\rho_{0}\right) \exp \left(\int_{0}^{t_{2}} C(\tau) d \tau\right)\right)\left(t_{2}-t_{1}\right),
\end{aligned}
$$

where $C(t)$ is given by (2.15), see also (2.12), and we applied Proposition 2 to estimate the total variation.

Approximating the characteristic function $\chi_{\left[t_{1}, t_{2}\right]}$ with a smooth function, and taking the limit, we still obtain the above estimate. This implies

$$
\begin{aligned}
& \left\|\rho^{N}\left(t_{1}, x\right)-\rho^{N}\left(t_{2}, x\right)\right\|_{\mathbf{L}^{\mathbf{1}}(\mathbb{R})} \\
& =\sup _{|\psi| \leq 1} \int_{\mathbb{R}}\left(\rho^{N}\left(t_{2}, x\right)-\rho^{N}\left(t_{1}, x\right)\right) \psi(x) d x \leq\left(t_{2}-t_{1}\right)\left(C\left(t_{2}\right)+v_{\max } T V\left(\rho_{0}\right) \exp \left(\int_{0}^{t_{2}} C(\tau) d \tau\right)\right) .
\end{aligned}
$$

Now we can apply [17, Theorem A.8] to conclude that there exists a sequence $\left\{N_{j}\right\}_{j=1}^{+\infty}, N_{j} \rightarrow+\infty$ as $j \rightarrow+\infty$, and a function $\rho$ such that

$$
\rho^{N_{j}} \rightarrow \rho \text { in } \mathbf{C}\left(\left[0,+\infty\left[; \mathbf{L}^{\mathbf{1}}(\mathbb{R})\right), \text { as } j \rightarrow+\infty \text { in } \mathbf{L}^{\mathbf{1}} l o c .\right.\right.
$$

In order to prove the limit $\lim _{N \rightarrow+\infty} q^{N}=q \in \mathbf{C}\left(\left[0,+\infty\left[; \mathbf{L}^{\mathbf{1}}(\mathbb{R})\right)\right.\right.$, in $\mathbf{L}^{\mathbf{1}}$ loc , we have to observe that according to [11, Proposition 1], we have

$$
\omega^{N}(t, x) \rightarrow \omega \in \mathbf{L}_{l o c}^{\infty}\left(\mathbb{R}^{+} \times \mathbb{R}\right) \text { as } N \rightarrow+\infty, \text { in } \mathbf{L}^{\mathbf{1}}{ }_{l o c} .
$$

In particular, this holds for every subsequence. In this way, considering (4.5), we can conclude that

$$
q^{N_{j}}=\omega^{N_{j}} \rho^{N_{j}} \rightarrow q=\omega \rho \text { in } \mathbf{C}\left(\left[0,+\infty\left[; \mathbf{L}^{\mathbf{1}}(\mathbb{R})\right), \text { as } j \rightarrow+\infty \text { in } \mathbf{L}^{\mathbf{1}}{ }_{l o c} .\right.\right.
$$

Therefore, the convergence of $\left(\rho^{N}, q^{N}\right)$ to $(\rho, q)$ up to a subsequence is ensured. Now we are left to show that the limit is a weak solution of (3.2). Let us define with some abuse of notation

$$
\left(K_{\eta} * V\right)^{N}(t, x):=\sum_{i=0}^{N-1} \sum_{k=0}^{N_{\eta}-1} \gamma_{i, j}(t) V_{i, j}(t) \chi_{\left[x_{i}(t), x_{i+1}(t)[\right.}(x) .
$$

We compute

$$
\begin{aligned}
\int_{0}^{+\infty} \int_{\mathbb{R}} \rho^{N}(t, x)\left(\begin{array}{c}
1 \\
\omega^{N}(t, x)
\end{array}\right)\left(K_{\eta} * V\right)^{N} \varphi_{x}(t, x) d x d t \\
=\int_{0}^{+\infty} \sum_{i=0}^{N-1} \rho_{i}(t)\left(\begin{array}{c}
1 \\
\omega_{i}
\end{array}\right) \dot{x}_{i}(t) \int_{x_{i}(t)}^{x_{i+1}(t)} \varphi_{x}(t, x) d x d t \\
=\int_{0}^{+\infty} \sum_{i=0}^{N-1} \rho_{i}(t)\left(\begin{array}{c}
1 \\
\omega_{i}
\end{array}\right) \dot{x}_{i}(t) D^{+}\left(\varphi_{i}(t)\right) d t
\end{aligned}
$$




$$
\begin{aligned}
& =-\int_{0}^{+\infty}\left[\sum_{i=0}^{N-2} D^{+}\left(\rho_{i}(t)\left(\begin{array}{c}
1 \\
\omega_{i}
\end{array}\right) \dot{x}_{i}(t)\right) \varphi_{i+1}(t)\right. \\
& \left.-\rho_{N-1}(t)\left(\begin{array}{c}
1 \\
\omega_{N-1}
\end{array}\right) \dot{x}_{N-1} \varphi_{N}(t)+\rho_{0}(t)\left(\begin{array}{c}
1 \\
\omega_{0}
\end{array}\right) \dot{x}_{0}(t) \varphi_{0}(t)\right] d t \\
& =-\int_{0}^{+\infty}\left\{\sum _ { i = 0 } ^ { N - 2 } \int _ { x _ { i } ( t ) } ^ { x _ { i + 1 } ( t ) } \left[\rho_{i}^{2}(t)\left(\begin{array}{c}
1 \\
\omega_{i}
\end{array}\right) N D^{+}\left(\dot{x}_{i}(t)\right) \varphi_{i+1}(t)\right.\right. \\
& \left.+\rho_{i}(t) N D^{+}\left(\rho_{i}\left(\begin{array}{c}
1 \\
\omega_{i}
\end{array}\right)\right) \dot{x}_{i+1} \varphi_{i+1}(t)\right] d x \\
& \left.-\rho_{N-1}(t)\left(\begin{array}{c}
1 \\
\omega_{N-1}
\end{array}\right) \dot{x}_{N-1}(t) \varphi_{N}(t)+\rho_{0}(t)\left(\begin{array}{c}
1 \\
\omega_{0}
\end{array}\right) \dot{x}_{0}(t) \varphi_{0}(t)\right\} d t .
\end{aligned}
$$

Following the calculations we used to obtain (4.4), we similarly get

$$
\begin{aligned}
& \int_{0}^{+\infty} \int_{\mathbb{R}} \rho^{N}(t, x)\left(\begin{array}{c}
1 \\
\omega^{N}(t, x)
\end{array}\right) \varphi_{t}(t, x) d x d t+\int_{\mathbb{R}} \rho^{N}(0, x)\left(\begin{array}{c}
1 \\
\omega^{N}(0, x)
\end{array}\right) \varphi(0, x) d x \\
& =\int_{0}^{+\infty}\left[\sum_{i=0}^{N-1} \int_{x_{i}(t)}^{x_{i+1}(t)} \rho_{i}^{2}(t)\left(\begin{array}{c}
1 \\
\omega_{i}
\end{array}\right) N\left(\dot{x}_{i+1}(t)-\dot{x}_{i}(t)\right) \varphi(t, x) d x\right. \\
& \left.+\sum_{i=0}^{N-2} D^{+}\left(\rho_{i}(t)\left(\begin{array}{c}
1 \\
\omega_{i}
\end{array}\right)\right) \dot{x}_{i+1}(t) \varphi_{i+1}(t)-\rho_{N-1}(t)\left(\begin{array}{c}
1 \\
\omega_{N-1}
\end{array}\right) \dot{x}_{N}(t) \varphi_{N}(t)+\rho_{0}(t)\left(\begin{array}{c}
1 \\
\omega_{0}
\end{array}\right) \dot{x}_{0}(t) \varphi_{0}(t)\right] d t .
\end{aligned}
$$

Then adding up both equations gives us

$$
\begin{gathered}
\left|\int_{0}^{+\infty} \int_{\mathbb{R}}\left(\rho^{N} \varphi_{t}+\rho^{N}\left(K_{\eta} * u\right)^{N} \varphi_{x}(t, x)\right)\left(\begin{array}{c}
1 \\
\omega^{N}
\end{array}\right) d x d t+\int_{\mathbb{R}} \rho^{N}(0, x)\left(\begin{array}{c}
1 \\
\omega^{N}(0, x)
\end{array}\right) \varphi(0, x) d x\right| \\
\quad=\left|\int_{0}^{+\infty}\left[\sum_{i=0}^{N-1} \rho_{i}^{2}\left(\begin{array}{c}
1 \\
\omega_{i}
\end{array}\right) N\left(\dot{x}_{i+1}(t)-\dot{x}_{i}(t)\right) \int_{x_{i}(t)}^{x_{i+1}(t)}\left(\varphi(t, x)-\varphi\left(t, x_{i+1}\right)\right) d x\right] d t\right| .
\end{gathered}
$$

Now we observe that

$$
\left|\int_{x_{i}(t)}^{x_{i+1}(t)}\left(\varphi(t, x)-\varphi\left(t, x_{i+1}\right)\right) d x\right| \leq\left\|\varphi_{x}(t, \cdot)\right\|_{\infty}\left(x_{i+1}(t)-x_{i}(t)\right)^{2} .
$$

Using this we obtain

$$
\begin{gathered}
\left\|\int_{0}^{+\infty} \int_{\mathbb{R}}\left(\rho^{N} \varphi_{t}+\rho^{N}\left(K_{\eta} * u\right)^{N} \varphi_{x}\right)\left(\begin{array}{c}
1 \\
\omega^{N}
\end{array}\right) d x d t+\int_{\mathbb{R}} \rho^{N}(0, x)\left(\begin{array}{c}
1 \\
\omega^{N}(0, x)
\end{array}\right) \varphi(0, x) d x\right\| \\
\quad \leq \int_{0}^{+\infty} \sum_{i=0}^{N-1} \frac{\max \left\{1, v_{\max }\right\}}{N^{2}} C(t)\left\|\varphi_{x}(t, \cdot)\right\|_{\infty} d t \leq \frac{\max \left\{1, v_{\max }\right\}}{N} T C(T) \sup _{t \in[0, T]}\left\|\varphi_{x}(t, \cdot)\right\|_{\infty},
\end{gathered}
$$


where $T>0$ is such that $\operatorname{supp}(\varphi) \subset[0, T] \times \mathbb{R}$. Obviously the right hand side converges to zero as $N \rightarrow+\infty$. In this way, we can conclude that $(\rho, q)$ is a weak solution.

Remark 3. We note that we have not only shown that the micro model (2.7) converges (up to a subsequence) to a weak solution of (3.2), but also existence of weak solutions of the macro model.

\section{$5 \quad$ Numerical simulations}

\section{$5.1 \quad$ Numerical schemes}

We shortly present the numerical tools used to solve the microscopic equations (2.7) and the macroscopic system (3.2).

Since the microscopic equations (2.7) are just a system of ordinary differential equations we solve this systems by solvers provided by MATLAB, e.g. ode45.

For the macroscopic equations, we recall that the system (3.2) is given by

$$
\left\{\begin{array}{l}
\rho_{t}+\left(\rho\left(K_{\eta} * V\left(\rho, \frac{q}{\rho}\right)\right)\right)_{x}=0 \\
q_{t}+\left(q\left(K_{\eta} * V\left(\rho, \frac{q}{\rho}\right)\right)\right)_{x}=0 .
\end{array}\right.
$$

Therefore, the two equations are only coupled via the non-local term, which has to be computed for each time step. The two equations can then be approximated separately. This leads to consider the upwind-type scheme introduced in [14]. The upwind flux is given by

$$
F_{j+\frac{1}{2}}^{n}=V_{j}^{n}\left(\begin{array}{c}
\rho_{j}^{n} \\
q_{j}^{n}
\end{array}\right)
$$

where

$$
V_{j}^{=} \sum_{k=0}^{\lfloor\eta / \Delta x\rfloor-1} \gamma_{k} V\left(\rho_{j+k+1},{ }^{n} \frac{q_{j+k+1}^{n}}{\rho_{j+k+1}^{n}}\right) \quad \text { and } \quad \gamma_{k}=\int_{k \Delta x}^{(k+1) \Delta x} K_{\eta}(y-x) d y .
$$

The complete scheme is then given by

$$
\left(\begin{array}{c}
\rho_{j}^{n+1} \\
q_{j}^{n+1}
\end{array}\right)=\left(\begin{array}{c}
\rho_{j}^{n} \\
q_{j}^{n}
\end{array}\right)-\lambda\left(F_{j+\frac{1}{2}}^{n}-F_{j-\frac{1}{2}}^{n}\right) .
$$

We note that this scheme is also in line with the Roe scheme presented in [25].

As CFL condition we choose an adaptive step size control determined by the maximum non-local velocity, i.e. $\Delta t^{n} \leq \Delta x / \max _{j} V_{j}^{n}$.

\subsection{Micro to macro convergence}

In this section we will numerically analyze the convergence of the micro dynamics (2.7) to the macro model (3.2).

Here and in the following we will restrict ourselves to the velocity function $V(\rho, \omega)=\omega-P(\rho)$ with $P(\rho)=6 \rho$. Therefore, we analyze a non-local version of the ARZ model. The initial conditions are Riemann initial conditions with the discontinuity being at $x=0$ and

$$
\rho_{l}=0.05, \quad \rho_{r}=0.05, \quad \omega_{l}=0.35, \quad \omega_{r}=0.8 .
$$




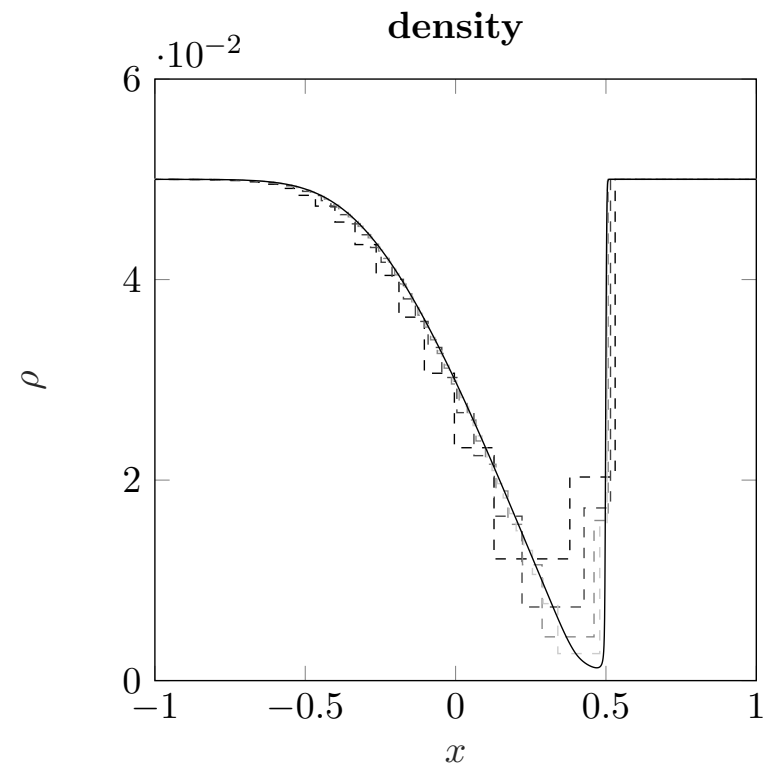

lagrangian marker

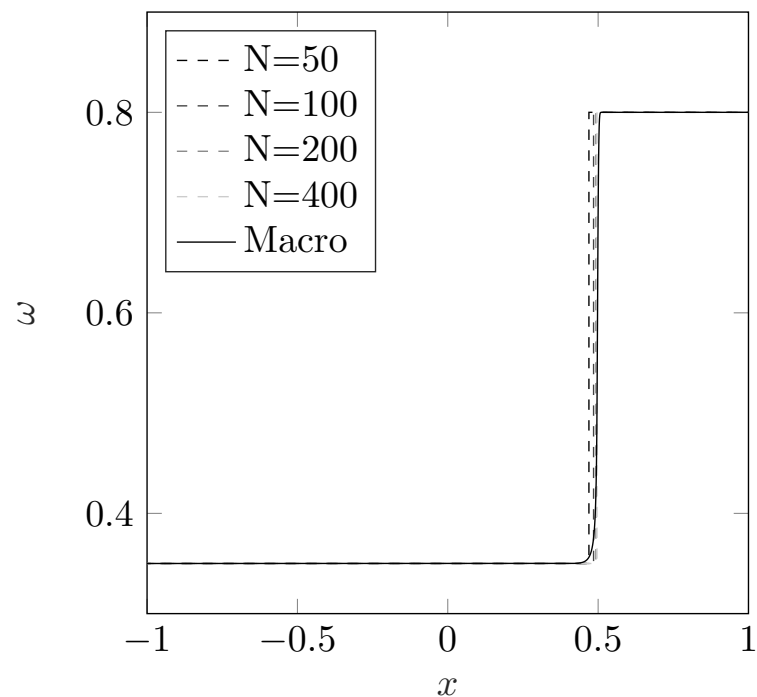

Figure 1: Solution of the microscopic for different $N$ and macroscopic model at $T=1$

Table 1: $\mathbf{L}^{\mathbf{1}}$ errors and convergence rate (c.r.) to a reference solution for the density $\rho$

\begin{tabular}{c|ccccccc}
$n$ & 0 & 1 & 2 & 3 & 4 & 5 & 6 \\
\hline $\mathbf{L}^{1}$-error & $3.30 \mathrm{e}-03$ & $4.90 \mathrm{e}-04$ & $3.16 \mathrm{e}-04$ & $2.05 \mathrm{e}-04$ & $1.31 \mathrm{e}-04$ & $8.09 \mathrm{e}-05$ & $4.52 \mathrm{e}-05$ \\
c.r. & - & 2.75 & 0.63 & 0.62 & 0.65 & 0.70 & 0.84
\end{tabular}

This test case corresponds to one of the examples presented in $[1,11]$.

We consider a linear kernel function and $\eta=0.1$ for our test cases. We are interested in the solution at the final time $T=1$.

In Figure 1, we can see the solution in the interval $[-1,1]$ for different numbers of vehicles and the macroscopic solution obtained by the scheme (5.1) for $\Delta x=10^{-3}$. The number $N$ corresponds to the number of vehicles initially distributed in the interval $[-1.5,1.5]$. In order to compare it to the macroscopic model, we consider a zoom on the interval $[-1,1]$. In addition, we set the velocity of the leading vehicle equal to $V(0.05,0.8)$, such that the leading car has the velocity given by the Riemann problem. As we can see the micro model converges to the solution of the macro model.

In addition, we analyze the convergence of the macroscopic scheme. Therefore, we compute a fine reference solution with $\Delta x=10^{-2} \cdot 2^{-8}$ and determine the experimental order of accuracy in the $\mathbf{L}^{1}$ norm for $\Delta x=10^{-2} \cdot 2^{-n}, n=0, \ldots, 6$. Due to the discontinuity of the Lagrangian marker we restrict ourselves to the density. The error terms can be seen in Table 1 and demonstrate that the numerical scheme (5.1) converges. In addition this justifies that we only consider the macro solution for a fixed and small $\Delta x$ in the micro to macro convergence above.

\subsection{Limits of the look ahead distance}

For non-local traffic flow models the limit for $\eta \rightarrow 0$ is of special interest, since the question of convergence to classical traffic flow models is still unanswered, see [8]. For first order traffic flow models, numerical examples suggest this convergence, see e.g. [14], but no general results can be proven, since the BV estimates blow up (see [20] for a proof in the case of monotone initial data). 


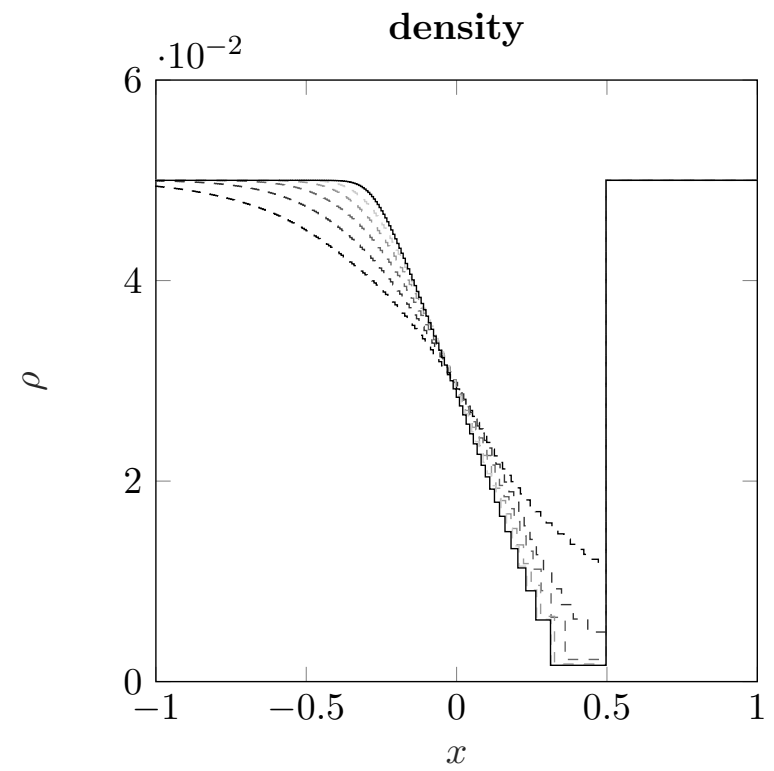

lagrangian marker

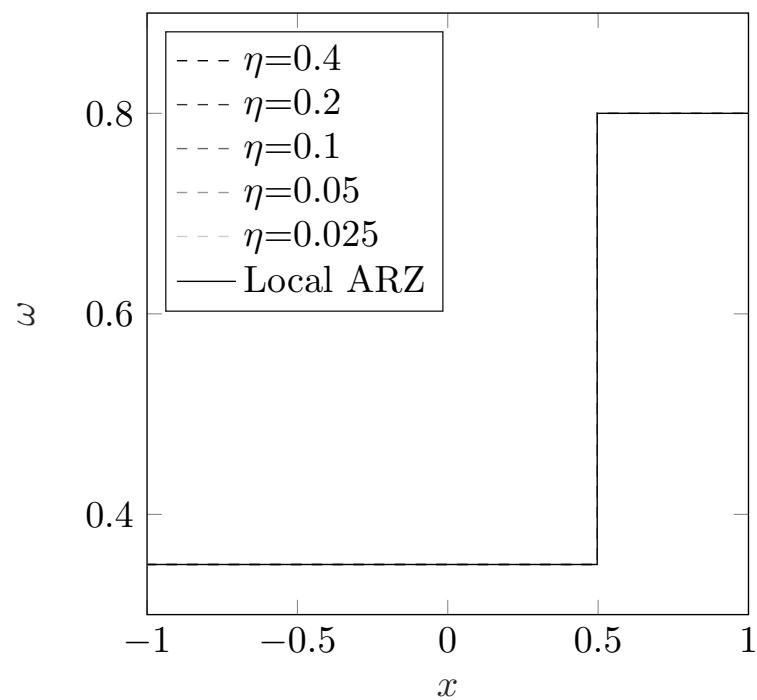

Figure 2: Convergence for $\eta \rightarrow 0$ of the microscopic model

Similarly, in the microscopic model, the estimate of Proposition 2 blows up for $\eta \rightarrow 0$. On the other hand for $\eta<1 / N$ the convergence is obvious since the non-local microscopic model is equivalent to the corresponding local model. For this reason, we consider $\eta>1 / N$ and $\eta \rightarrow 0$ for a fixed $N$ in the following and compare it to the local model. In addition, we show the behavior of the macroscopic model for $\eta \rightarrow 0$.

We consider $N=500, \Delta x=10^{-4}$ and $\eta \in\{0.4,0.2,0.1,0.05,0.025\}$. Figures 2 and 3 show the results for the microscopic and macroscopic model, respectively. As can be seen in Figure 2, the convergence can be observed for both the Lagrangian marker and the density for $\eta \rightarrow 0$. The solution of the microscopic ARZ model is computed by using the atomization scheme presented in [11, Section 3]. In order to obtain the solution of the ARZ model, we use the Godunov scheme presented in [21]. Similarly to the microscopic case, the macroscopic model seems to converge to the solution of the ARZ model, see Figure 3.

Another interesting limit is the case $\eta \rightarrow+\infty$ as analyzed in [6] for a first order model. In contrast to the limit $\eta \rightarrow 0$, the BV estimates in Proposition 2 are still valid. Especially the $C(t)$ in (2.15) converges to 0 for $\eta \rightarrow+\infty$, such that the classical estimate is obtained. In order to study the convergence, we consider now an initial condition with compact support, i.e.

$$
\rho_{0}(x)=0.05 \cdot \mathbb{1}_{[-0.5,0.5]}(x) .
$$

Due to the high computational cost, we consider $\Delta x=10^{-2}$ and $N=100$ for the simulations. The empty road speed outside the compact support of the density is assumed to be the velocity of the leading vehicle.

The Lagrangian marker exhibits the same discontinuity as in the examples above. In the first order case, non-local traffic flow models tend to a classical LWR equation with maximum velocity. A very similar convergence result can be obtained for both the microscopic (Figure 4) and macroscopic level (Figure 5). We note that, in Figures 4 and 5 , the $x$-axis are different, since the microscopic density cannot be computed outside the compact support of the solution. In order to get more meaningful figures, we zoom in for the microscopic solution. We obtain that the model converges to the transport equation with the velocity given by the Lagrangian marker for $x>0$, i.e. $\omega_{r}=0.8$. 

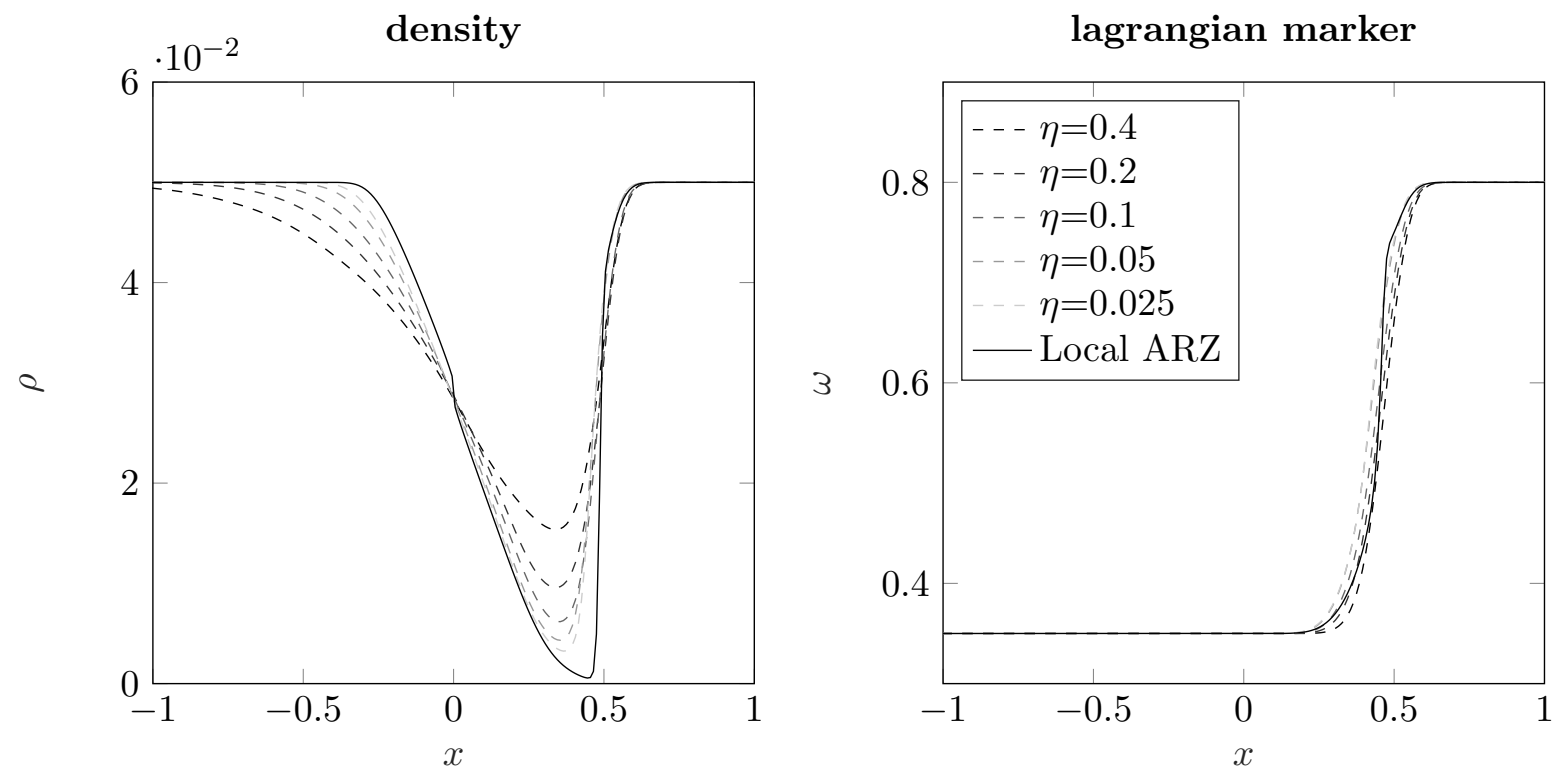

Figure 3: Convergence for $\eta \rightarrow 0$ of the macroscopic model
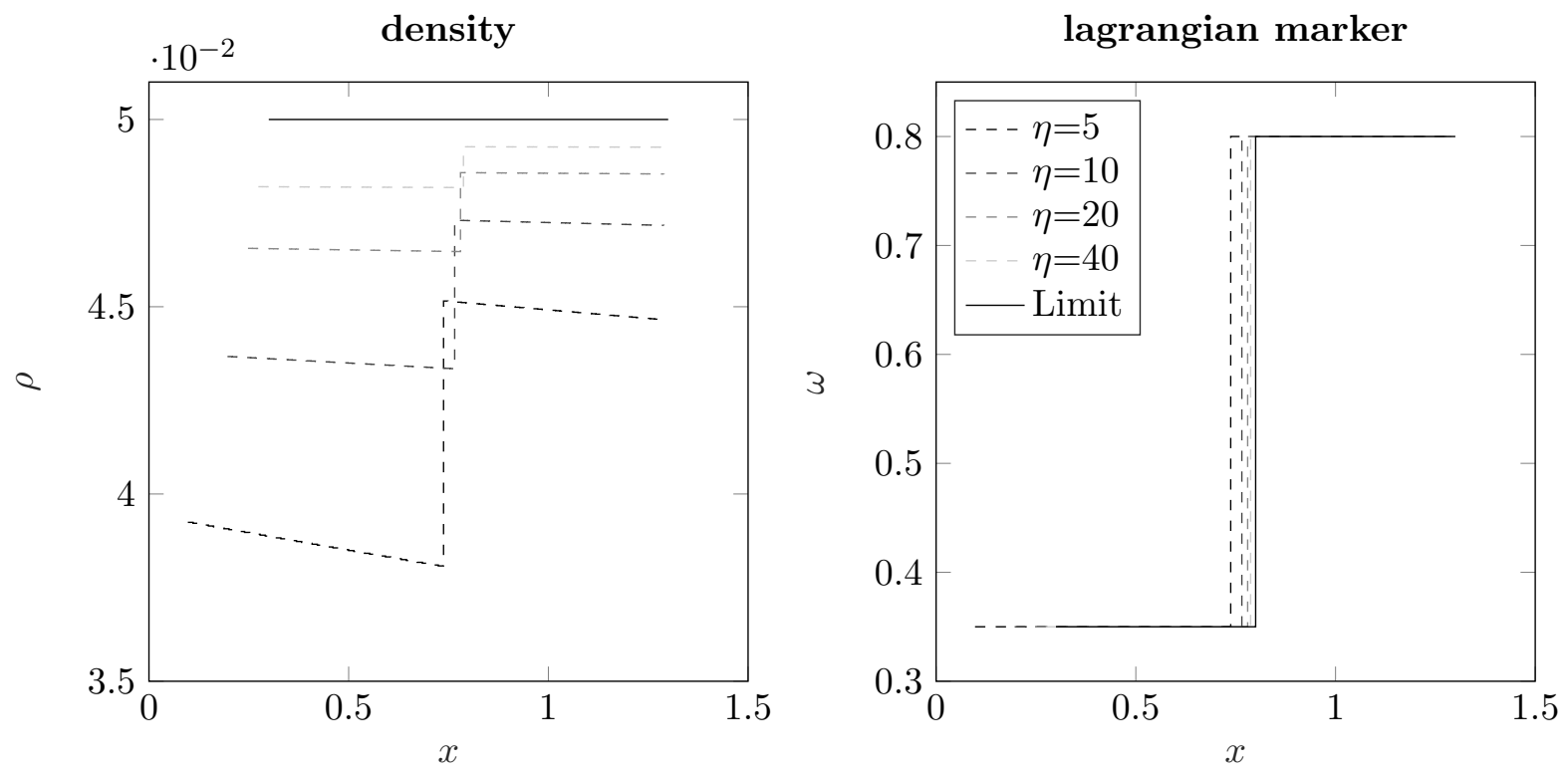

Figure 4: Convergence for $\eta \rightarrow \infty$ of the microscopic model

\section{Conclusion}

Inspired by a first order microscopic model for non-local traffic flow and by the second order macroscopic GARZ traffic model, we derived a second order microscopic non-local traffic model. This model inherits a maximum principle. Under suitable assumptions on the initial condition, we proved that the corresponding discrete density function has bounded total variation. Therefore, we could prove the convergence to a weak solution of a new macroscopic non-local second order GARZ model, for which we thus prove existence of weak solutions. Numerical examples sustain the theoretical results about the micro to macro convergence. In addition, we show the behavior of the 

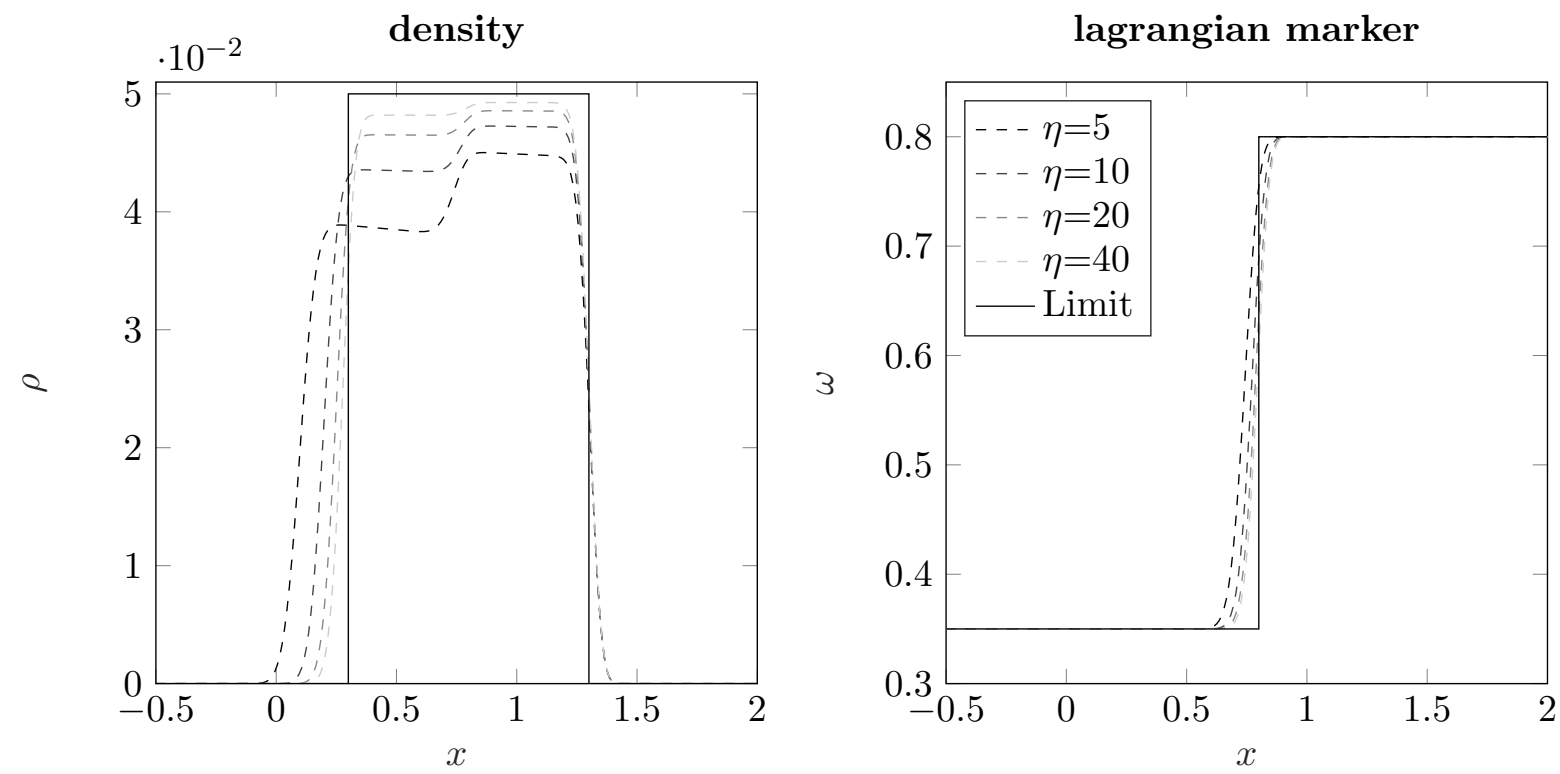

Figure 5: Convergence for $\eta \rightarrow+\infty$ of the macroscopic model

micro and macros models when the look-ahead distance goes to zero. Similarly to the first order case, the model seems to converge to the corresponding local model. If the look-ahead distance goes to infinity under suitable assumptions on the Lagrangian marker, the model seems to converge to the solution of two linear transport equation.

Future works will include a deeper analysis of the second order non-local GARZ model and extensions to networks.

\section{Acknowledgements}

This work was financially supported by the project "Non-local conservation laws for engineering applications" co-funded by DAAD (Project-ID 57445223) and the PHC Procope (Project no. 42503RM).

\section{References}

[1] A. Aw, A. Klar, T. Materne, and M. Rascle. Derivation of continuum traffic flow models from microscopic follow-the-leader models. SIAM J. Appl. Math., 63(1):259-278, 2002.

[2] A. Aw and M. Rascle. Resurrection of "second order" models of traffic flow. SIAM J. Appl. Math., 60(3):916-938, 2000.

[3] S. Blandin and P. Goatin. Well-posedness of a conservation law with non-local flux arising in traffic flow modeling. Numer. Math., 132(2):217-241, 2016.

[4] M. Burger, S. Göttlich, and T. Jung. Derivation of a first order traffic flow model of Lighthill-WhithamRichards type. IFAC-PapersOnLine, 51(9):49 - 54, 2018. 15th IFAC Symposium on Control in Transportation Systems CTS 2018.

[5] F. A. Chiarello, J. Friedrich, P. Goatin, S. Göttlich, and O. Kolb. A non-local traffic flow model for 1-to-1 junctions. European Journal Appl. Math., page 121, May 2019. 
[6] F. A. Chiarello and P. Goatin. Global entropy weak solutions for general non-local traffic flow models with anisotropic kernel. ESAIM Math. Model. Numer. Anal., 52(1):163-180, 2018.

[7] J. Chien and W. Shen. Stationary wave profiles for nonlocal particle models of traffic flow on rough roads. NoDEA Nonlinear Differential Equations Appl., 26(6):Paper No. 53, 2019.

[8] M. Colombo, G. Crippa, M. Graff, and L. V. Spinolo. Recent results on the singular local limit for nonlocal conservation laws, 2019.

[9] R. M. Colombo and E. Rossi. On the micro-macro limit in traffic flow. Rend. Semin. Mat. Univ. Padova, 131:217-235, 2014.

[10] M. Di Francesco, S. Fagioli, and E. Radici. Deterministic particle approximation for nonlocal transport equations with nonlinear mobility. J. Differential Equations, 266(5):2830-2868, 2019.

[11] M. di Francesco, S. Fagioli, and M. D. Rosini. Many particle approximation of the Aw-Rascle-Zhang second order model for vehicular traffic. Math. Biosci. Eng., 14(1):127-141, 2017.

[12] M. Di Francesco and M. D. Rosini. Rigorous derivation of nonlinear scalar conservation laws from follow-the-leader type models via many particle limit. Arch. Ration. Mech. Anal., 217(3):831-871, 2015.

[13] S. Fan, M. Herty, and B. Seibold. Comparative model accuracy of a data-fitted generalized Aw-RascleZhang model. Netw. Heterog. Media, 9(2):239-268, 2014.

[14] J. Friedrich, O. Kolb, and S. Göttlich. A Godunov type scheme for a class of LWR traffic flow models with non-local flux. Netw. Heterog. Media, 13(4):531-547, 2018.

[15] D. C. Gazis, R. Herman, and R. W. Rothery. Nonlinear follow-the-leader models of traffic flow. Operations Res., 9:545-567, 1961.

[16] P. Goatin and F. Rossi. A traffic flow model with non-smooth metric interaction: well-posedness and micro-macro limit. Commun. Math. Sci., 15(1):261-287, 2017.

[17] H. Holden and N. H. Risebro. Front tracking for hyperbolic conservation laws, volume 152 of Applied Mathematical Sciences. Springer, Heidelberg, second edition, 2015.

[18] H. Holden and N. H. Risebro. The continuum limit of Follow-the-Leader models - a short proof. Discrete Contin. Dyn. Syst., 38(2):715-722, 2018.

[19] H. Holden and N. H. Risebro. Follow-the-leader models can be viewed as a numerical approximation to the Lighthill-Whitham-Richards model for traffic flow. Netw. Heterog. Media, 13(3):409-421, 2018.

[20] A. Keimer and L. Pflug. On approximation of local conservation laws by nonlocal conservation laws. $J$. Math. Anal. Appl., 475(2):1927-1955, 2019.

[21] O. Kolb, S. Göttlich, and P. Goatin. Capacity drop and traffic control for a second order traffic model. Netw. Heterog. Media, 12(4):663-681, 2017.

[22] M. J. Lighthill and G. B. Whitham. On kinematic waves. II. A theory of traffic flow on long crowded roads. Proc. Roy. Soc. London Ser. A, 229:317-345, 1955.

[23] P. I. Richards. Shock waves on the highway. Operations Res., 4:42-51, 1956.

[24] J. Ridder and W. Shen. Traveling waves for nonlocal models of traffic flow. Discrete Contin. Dyn. Syst., 39(7):4001-4040, 2019.

[25] P. L. Roe. Approximate Riemann solvers, parameter vectors, and difference schemes. Journal of computational physics, 43(2):357-372, 1981.

[26] H. Zhang. A non-equilibrium traffic model devoid of gas-like behavior. Transportation Research Part B: Methodological, 36(3):275 - 290, 2002. 